\title{
Multifractal Formalism in Tasks of Dynamic Plasticity
}

\author{
Aleksander Kuznetsov ${ }^{1}$, Georgii Savenkov ${ }^{1,2,{ }^{*}}$, and Vladimir Stolyarov ${ }^{3}$ \\ ${ }^{1}$ JSC «Armalit Mechanical Engineering Plant»,198097 St. Petersburg, Russia \\ ${ }^{2}$ Saint Petersburg State Technological Institute (technical university), 190013 St. Petersburg, Russia \\ ${ }^{3}$ Mechanical Engineering Research Institute of RAS, 101990, Moscow, Russia
}

\begin{abstract}
The application of the multifractal parameterization method for comparing structural changes observed in an optical microscope on steel St.3 subjected to severe plastic deformation and high-rate superplastic deformation is investigated.
\end{abstract}

\section{Introduction}

In recent years, the attention of many researchers has been focused on the features of processes under conditions of extreme deformation, which has received the terms "high", "large", "intense", "megaplastic" [1 - 3]. Of no less interest is superplastic deformation associated with the problem of forming [4]. For both deformation types, there is a special mode, the most interesting and least studied. This mode is associated with a high strain rate $\left(>1 \mathrm{~s}^{-1}\right)$, at which superplasticity should not appear at all. The modes and model of superplastic deformation at rates of $10^{0}-10^{5} \mathrm{~s}^{-1}$ are considered in $[5,6]$. However, this was done for structural superplasticity, which manifests itself under certain conditions:

- strain temperature $T \sim 0.5 T_{m}$ and higher (Tm - melting point);

- presence ultrafine grains $\left(d_{z} \leq 10 \mu \mathrm{m}\right)$ in the structure, stable in the temperature range $T=$ (0.4 - 0.6) $T_{m}$.

Intensive plastic deformation can also be carried out with the dynamic method of obtaining nanostructured materials. In this case, the materials are subjected to high-rate deformation in a pulsed mode by the method of dynamic equal-channel angular pressing (ECAP) to obtain nanostructured materials due to the energy of compressed gases from the combustion products of gunpowder [7]. An interesting question arises: is there any similarity between these two types of high-rate deformations and what is it? The answer to these questions is possible if experiments with the same material and with the same strain rate are available. Taking into account the rates range, such experiments are rather difficult to carry out. However, there is an area of physics that allows such experiments to be carried out. This area of explosion physics is related to the processes of formation and tension of cumulative jets $\left(\mathrm{CJ}_{\mathrm{s}}\right)$ and their penetration into an obstacle. When forming and tension the $\mathrm{CJ}_{\mathrm{s}}$, superplastic deformation is manifested, and during melting, severe deformation, which can reach $100-1000 \%$, if we correlate it with the inclination of the reference grain lines in the direction of rolling [8].

\footnotetext{
${ }^{*}$ Corresponding author: sav-georgij@yandex.ru
} 
The aim of this work is to identify the features of the of intense and superplastic deformations processes under conditions of high-rate $\left(>10^{4} \mathrm{~s}^{-1}\right)$ deformation of steel samples by quantitative characterization of the created structure images.

\section{Experimental}

\subsection{Material and methods}

Two samples of low-carbon steel St. 3 with an average grain size of $35 \mu \mathrm{m}$ were investigated. Sample 1 was an engaging tail element of a plane cumulative jet (the so-called "wrecker" with jet elements), which was part of a cumulative knife formed as a result of compression of a steel angle element during an explosion (limited strain $\varepsilon=300-400 \%$ ). Since a plane cumulative jet is considered, the strain rate was $\dot{\varepsilon}=5 \cdot\left(10^{3}-10^{4}\right) \mathrm{s}^{-1}$. Sample 2 with initial grain size of $40 \mu \mathrm{m}$ in the disk shape of $\varnothing 90 \times 10 \mathrm{~mm}$ was subjected to severe plastic deformation by plane cumulative jet. The initial impact velocity of the jet head is $\mathrm{V}_{0} \sim 3.5$ $\mathrm{km} / \mathrm{s}$. The structure was investigated on optical microscopes "Affri" and "Aksiovert". The cross-sectional planes were chosen so that they were parallel to the loading direction and perpendicular to the cumulative knife cross-section plane. In the experiments, each sample was a thermodynamically unbalanced physical multilevel system with nonlinear properties, in which the structural-deformation states are determined by the statistical-thermodynamic nature of the deformed metal and the symmetry of the crystal structure. Therefore, when processing images of structures, the multifractal parameterization (MFP) method was used, that allows analyzing images with signs of a scale (structural) hierarchy [9].

The MFP method is based on the construction of a measures set that approximates the structure under study. By dividing the original image into cells, it is possible to assign each cell its own measure (weight), which corresponds to the nature of the object: mass, square, energy, etc. The task solution is implemented in the original Fractal program [9]. The analysis procedure is as follows. Initially, the original gray scale image approaches black and white (binary) representation. The binarization operation is necessary for the subsequent analysis of information using a computer that processes impulse signals. Binary representations are collections of elementary sections (pixels). For example, elementary areas - pixels that fall on the analyzed objects are designated "1" and become "white". Pixels that do not fall on the studied structural elements are indicated by the number " 0 " and become "black".

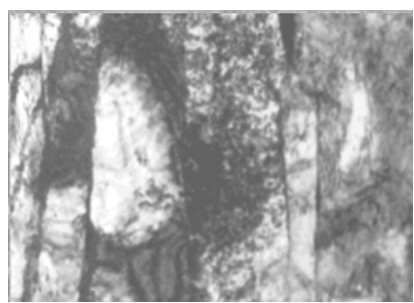

a

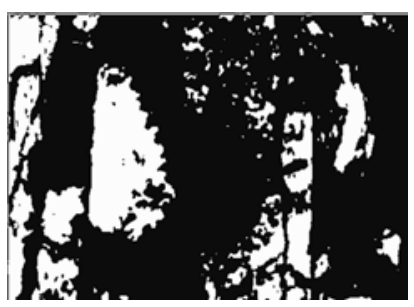

b

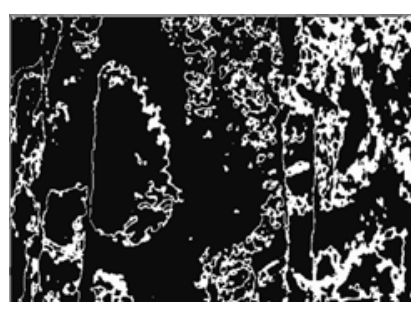

c

Fig. 1. Bainite-martensitic structure of steel on a translucent screen of an electron microscope (x34000): a - the original gray scale image in 256 shades; b - binary representation of twodimensional objects analyzed after one-step binarization; c - representation of linear objects (contours) using a slot after 2-step binarization [10].

Binarization is an important operation in analysis, an example of which is shown in Fig. 1. If the original gray scale image is 256-gray tones and is composed of flat (2-dimensional) 
objects to be analyzed, then to obtain a black-and-white image, it is sufficient to select onestep binarization, the implementation of which will ensure the best coincidence of the gray scale image objects contours with the black and white representation. If it is necessary to analyze linear objects (contours), then two-step binarization can be implemented, which will provide a representation with the best approximation to the analyzed image. Then virtual scale networks with cells of different sizes $2 \times 2,3 \times 3,4 \times 4$, etc. are lowered onto the binary representation. The number of cells corresponding to the "white" and "black" regions is divided by the number of all pixels showing the representation of the structure, and therefore a measure for each pixel is derived.

On this basis, the measures of the coarse decomposition of the image in large cells (which consist of $2 \times 2,3 \times 3$, etc. pixels) are generated by simple addition of the measures. Thus, a string of equal-cell decompositions is obtained with the sizes of cells from the given set $\left\{1_{\mathrm{k}}\right\}$ and with measures $\left\{\mu_{\mathrm{ik}}, \mathrm{I}=1, \ldots, \mathrm{N}_{\mathrm{k}}\right\}$, determined by the given measures of unit cells. For each partition into larger cells of size $l_{k} \times l_{k}$, a characteristic measure is constructed as the distribution of equal-cell units $\mu_{\mathrm{i}}\left(\mu_{\mathrm{i}}=\mathrm{M}_{\mathrm{i}} / \Sigma \mathrm{M}_{\mathrm{i}}\right.$, where $\mathrm{M}_{\mathrm{i}}$ is the number of units in the $\mathrm{i}$ large cell, $\Sigma \mathrm{M}_{\mathrm{i}}$ is the sum of units in the matrix of large cells, $\left.\mathrm{i}=1,2,3, \ldots, \mathrm{N}\right)$. Then, to select the values of $\mathrm{q}$ from a certain range of dimensions $[-200 \div+200]$, multifractal characteristics of $f(\alpha)$-spectra and $\mathrm{D}_{\mathrm{q}}$-spectra of Renyi dimensions are calculated (Fig. 2).

\subsection{Some functions and parameters of multifractal analysis}

The physical meaning of the function $f(\alpha)$ is the dimension of a homogeneous fractal subset from a given (initial) set of black and white pixels, characterized by similar probabilities of filling the cells. The selection of the function $f(\alpha)$ values for different $\alpha$ is the spectrum of fractal dimensions of homogeneous subsets into which the initial set can be divided ( $\alpha$ is a scaling exponent, known in mathematics as the Lipschitz - Hölder exponent [11]).
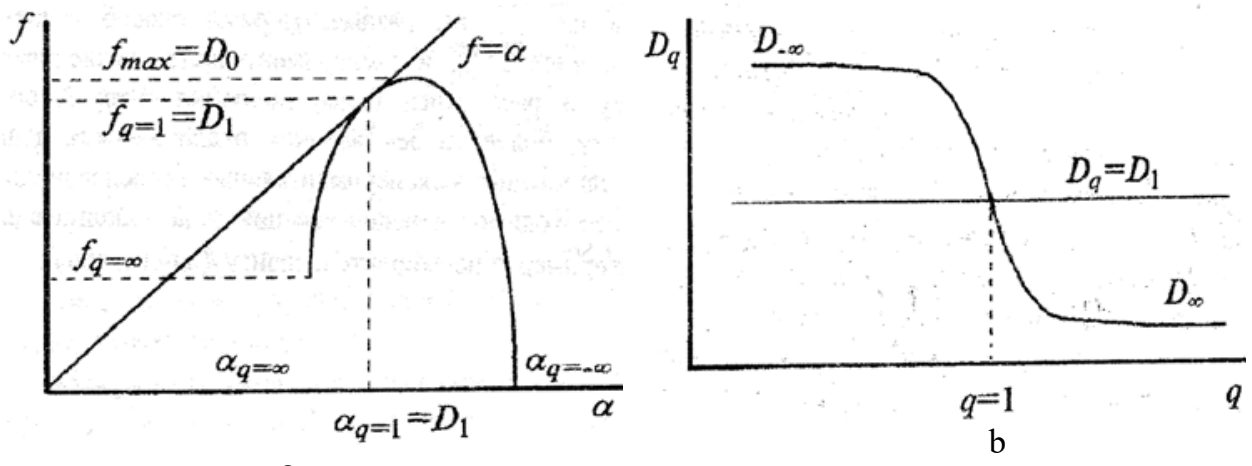

a

Fig. 2. The main dependences of MFP: a - $f(\alpha)$ spectrum; $\mathrm{b}-D_{q}$ - spectrum Renyi.

The probabilistic behavior of the fractal measure of multifractalism is described by the generalized statistical sum $Z(q, l)$ through the index $q$, determined by the coefficient of the multifractal moment $i$, which can be equal to any real number. Generalized statistical sum:

$$
Z(q, l)=\sum p_{i}^{q}(l) \sim l^{\tau(q)},
$$

where: $-\infty<q<\infty$. In this expression, the summation occurs over all $i$ from 1 to $\mathrm{N}(l)$.

The spectrum of the generalized Renyi dimension:

$$
D_{q}=\tau(q) /(q-1),
$$


where $\tau(q)=\lim _{l \rightarrow 0}[\ln Z(q, l) / \ln l]$ - non-linear function that characterizes the distribution of points (pixels) within the initial setting. The latter is found by numerical differentiation with respect to the slope of the experimental dependence $\ln Z(q, l)$. The sought multifractal is characterized by the graphical dependence of the generalized partition function $Z(q, l)$ as $l \rightarrow 0$. If $D_{q}=$ const, (does not depend on q), then the derived set is a regular fractal with dimension $D$. If $D_{q}$ depends on $q$, then the set under consideration is a multifractal. Characteristics $D_{q}$ are informative for positive values of $q$, since for $q \gg>1$ the values of $D_{q}$ correspond to higher values of entropy.

The results of the "Fractal" program are displayed on the monitor screen with the display of the original image, its binary display, the spectrum of generalized dimensions $D_{q}$ and a set of multifractal parameters (Fig. 3): $D_{0 f}$ is the spatial (Hausdorf) dimension of the set.
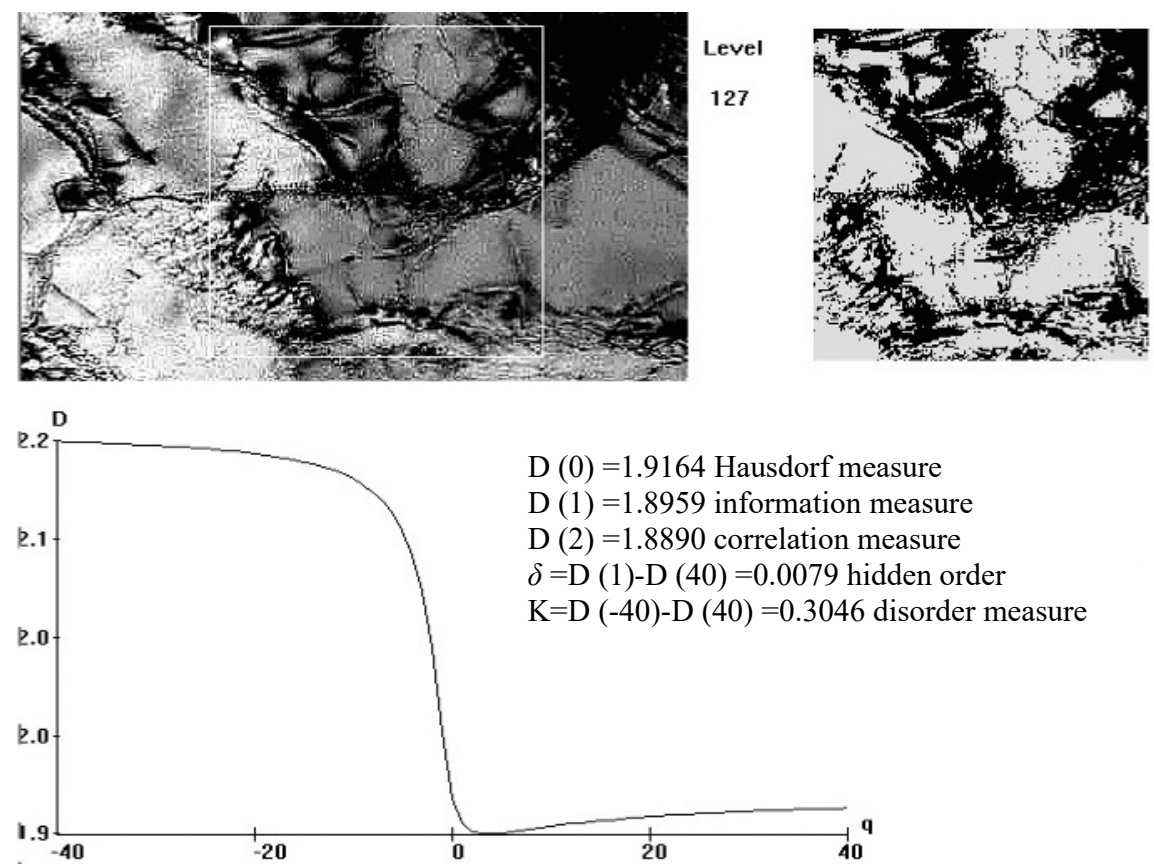

Fig. 3. The cycle of the "Fractal" program with calculations results and the dimensions spectrum on the computer monitor. The white frame shows the analyzed sector of the original halftone image converted to binary representation.

Evaluation of its geometric image, but it is a very approximate characteristic of a multifractal, since it does not carry any information about the statistical properties of the object. From the materials science point of view, this parameter is sensitive to the relief of grain boundaries and characterizes the tortuosity (relief) of the areas contours selected for analysis. $D_{l}$ is the information dimension associated with the entropy $S(l)$ of the set under consideration by the relation $D_{l}=-\lim [\mathrm{S}(l) / \ln l]$ as $l \rightarrow 0$. Here entropy $\mathrm{S}$ is a measure of the information amount required to determine the system state; $D_{2}$ correlation size characterizing the integral dependence of the correlating set on the cell sizes 1 at $1 \rightarrow$ $0 ; \boldsymbol{\delta}=D_{1}-D_{q}-$ non-negative measure of order (hidden order) and symmetry breaking for the general configuration of the structure under study as a whole. The larger the value of $\delta$ (in absolute value), the more ordered the structure and contains a large periodic component. The disappearance of $\delta$ represents an increase in chaos in the system of representative points and is a sign of an approaching structure-phase transition (TFT) in the structure of 
a real material ( $\delta=0$ means that SPT has occurred). $\boldsymbol{K}=D_{-q}-D_{+q}$ is the homogeneity parameter characterizing the distribution of individual objects in the structure under study in the surrounding (Euclidean) space, the higher the $\mathrm{K}$ value, the more homogeneous the structure.

The above multifractal parameters allow quantitative calibration of the processed images. That is, they are structural information criteria for searching for correlations of quantitative parameters of the obtained structure images with structurally determined properties (mechanical, magnetic, corrosion, etc.) of the material. For more details on the measures $\left\{\mu_{\mathrm{ik}}, I=1, \ldots \mathrm{N}_{\mathrm{k}}\right\}$, the functions $f(\alpha)$, and the spectrum of Renyi dimensions $D_{q}$, see [12].

From the statistical range of multifractal parameters, in this work, we use the spatial dimension of the object $D_{0 f}$ and the hidden order $\delta$.

\section{Results and discussion}

\subsection{Pestle and jet}

Consequence of change of structural changes, starting from end fragments of pestle and jet is shown on fig. $4-8$. Thus on the pic. 4 a the pestle's element structure is shown which is the closest to the jet's tail. It is visible that the pestle fragment is actually cut through by lengthy (up to $0,5 \mathrm{~mm}$ ) Luders bands of $\sim 80 \mathrm{mcm}$ width. Sequential changes in the structure, starting from the end fragments of the pestle and the jet, are shown in Fig. 4 - 8. In fig. 4a, it can be seen that the structure of the element closest to the jet tail is cut by long (up to 0.5 mm) Luders strips up to $80 \mu \mathrm{m}$ wide.

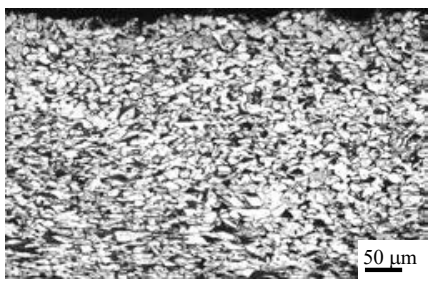

a

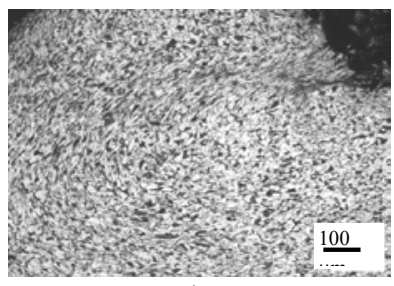

b

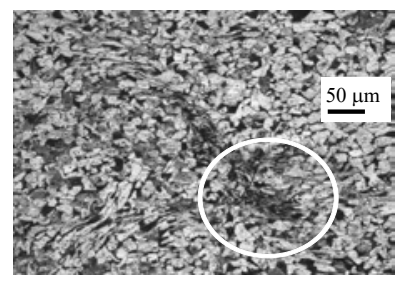

C

Fig. 4. Microstructure of pestle and jet areas with Luders stripes (a), vertical view (b, c) (the crack nucleus is shown in the circle).

With increasing distance from the end of the jet, a vortex structure arises, consisting of adiabatic shear bands (SBs) with a width of less than $10 \mu \mathrm{m}$. The dimensions of the vortex regions are from 50 to $300 \mu \mathrm{m}$ (Fig. 4 b, c), cracks appear between the swirling structures (Fig. 4c). Also, with distance from the tail of the jet, separate branching SBs of different lengths (Fig. 5a) and shape (Fig. 5b) appear.

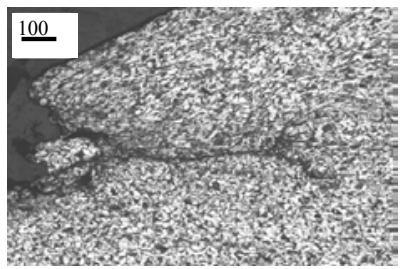

a

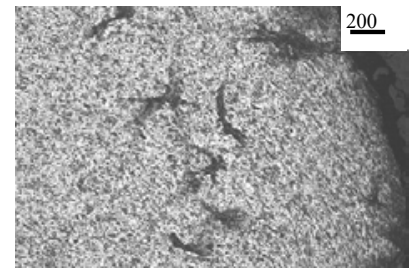

b

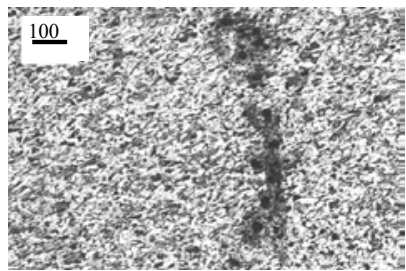

C

Fig. 5. Adiabatic shear bands in jet sections. 
Pores are formed along the bands (Fig. 5c), which can be seen already in the regions outside the adiabatic shear bands (Fig. 6). It follows from the structure images that the part of the pestle body distant from the tail (almost narrower than the jet) consists of dynamically polygonized grains, the initial grain size decreases, and grain shape becomes equiaxed. Thus, it can be assumed that in the tension process of a plane cumulative jet, the material was deformed with negligible strain hardening under conditions of warm or hot deformation. This activates rotational deformation modes or vortices. Their inclusion in the general deformation leads to the appearance of additional degrees of freedom of structure elements on which elastic energy can be dissipated [1]. Thus, it can be assumed that in the tension process of a plane cumulative jet, the material was deformed with negligible strain hardening under conditions of warm or hot deformation. This activates rotational deformation modes or vortices. Their inclusion in the general deformation leads to the appearance of additional degrees of freedom of structure elements on which elastic energy can be dissipated [1].

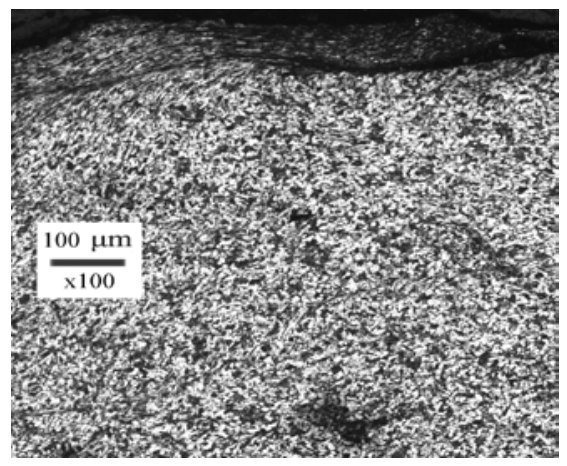

a

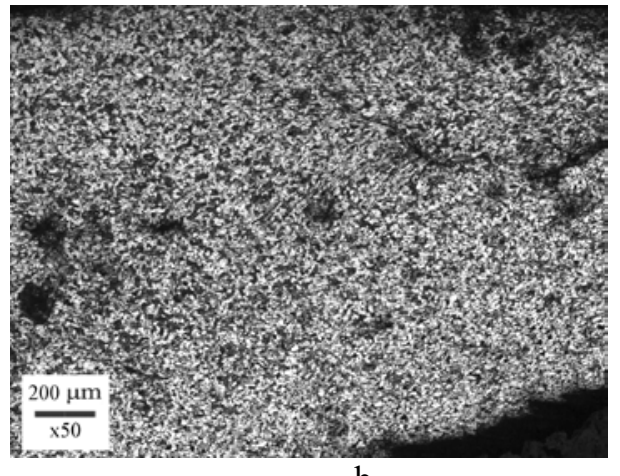

b

Fig. 6. Porosity in areas far from the end elements of the jet.

Multifractal analysis showed that structural changes correlate with the parameter $\delta$ and are carried out in the following order: 0.09 (extended bands of adiabatic shear) $\rightarrow 0.048$ (localized flow) $\rightarrow 0.036$ (localized eddies) $\rightarrow 0.025$ (dynamic polygonization $\rightarrow$ beginning of the structural phase transition) (fig. 7). The following picture can be traced by the parameter $D_{0 f}$. In the end part of the pestle, in the zones of plastic flow, the grains are deformed and unequal with an axial ratio of more than 1:10. This fact is quite remarkable. Due to SPD, the grains do not repeat the deformation of the entire sample, their elongation in the direction of tension is insignificant in comparison with the elongation of the sample [13]. This fact determines the decisive contribution of SPD to grain boundary sliding due to the involvement of a macroscopically extended system of conjugate boundaries in the overall process along which coherent sliding occurs.

In our case, the contribution of grain deformation, although at the last stage of jet tension, is very large and amounts to about $25 \%$ of the total deformation. With such a grain structure, the "average" filling of the image field is characterized by a measure of $D_{\text {of }}<1.93$. When $\delta$ approaches 0.04 , the $D_{0 f}$ increases to 1.95 . This indicates a structure refinement and the formation of new equiaxed grains and subgrains as a result of dynamic polygonization. The onset of the structure-phase transformation is accompanied by an increase in $D_{0 f}$ to 1.96 , a decrease in $\delta$ to 0.025 , a change in the flow curve of the medium, and recrystallization. Although no signs of dynamic recrystallization were found, the latter can be considered a less likely event. The sequence of changes in the parameters $\delta$ and $D_{0 f}$ does not contradict the results of hot deformation at rates up to $10^{5} \mathrm{~s}^{-1}$ [14-15]. It can be assumed that plastic deformation during the formation and extension of the cumulative knife leads to a local and 
then volumetric structure-phase transformation, which manifests itself in the refinement of dynamically polygonized grains.
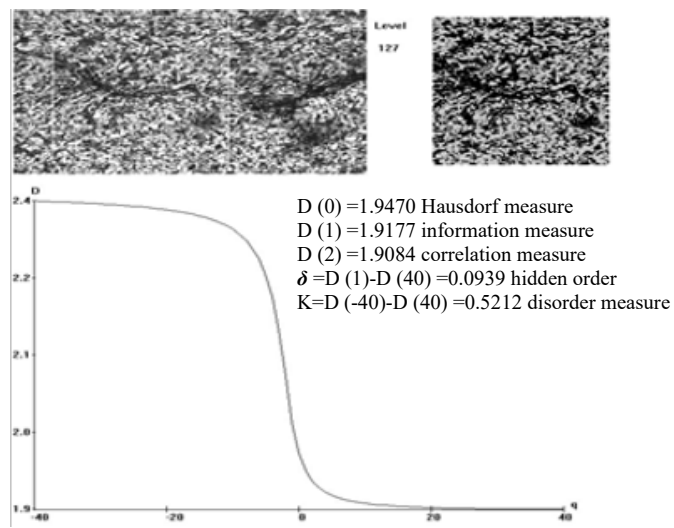

a)

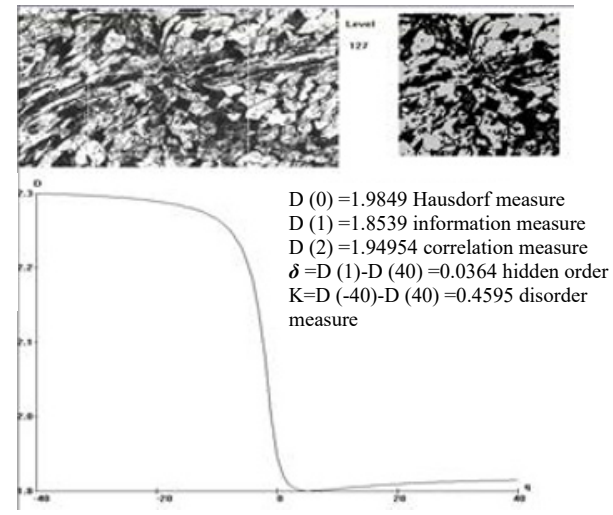

b)

Fig. 7. Images on the computer screen when using the Fractal program.

It can be assumed that the latent energy accumulated in the process of grain recovery served as the driving force of the reaction, and the mechanism of grain boundary sliding played a dominant role throughout most of the jet tension process during the implementation of superplastic deformation.

\subsection{Target}

For the study, an area was selected at a distance of $\sim 1 / 3$ from the frontal surface, where the stationary penetration stage of starts steadily [16]. The structure of this barrier section is shown in Fig. 8. SPD of the target material results in flows of non-equiaxed grains elongated in the direction of maximum tensile stresses. The streams are shown as binary representations of the original grayscale images of structures obtained at different binarization thresholds (Fig. 9). The overlap regions that have arisen during the structural relaxation of the material, with the same complex flow patterns of oriented grains, characterize dissipative structures of a nonequilibrium state (DSNS).

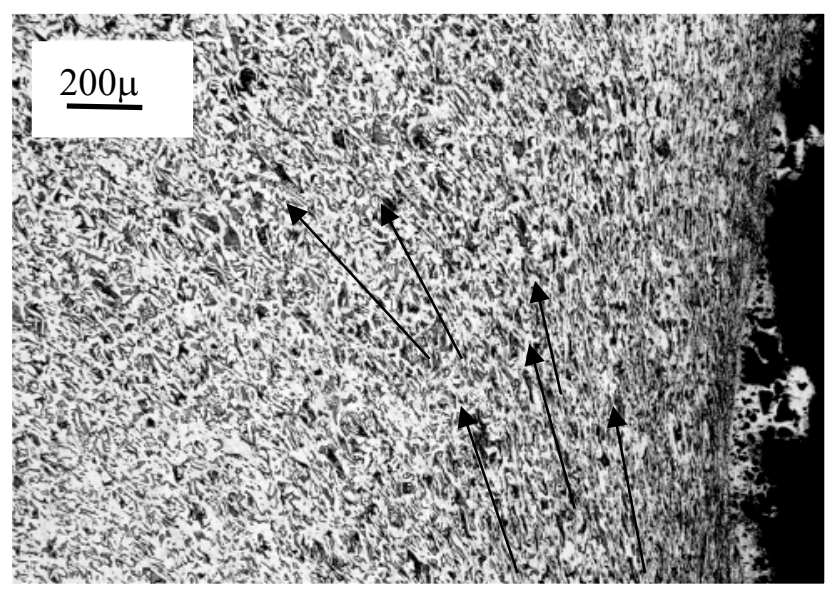

Fig. 8. Target structure (arrows indicate traces of nonequilibrium structural states). 
Traces of the DSNS, as seen in Fig. 9, are distinguished by the contours of localized elongated sections, which consist of grains of similar morphology, surrounded by chaotically oriented grains. These traces can also be attributed to "static autostructures", spatially oriented localized grain formations. Since DSNSs consist of macroscopic objects (grains of similar morphology), and not of dislocation ensembles of the mesoscopic range, they are larger than the fracture bands and BAS.

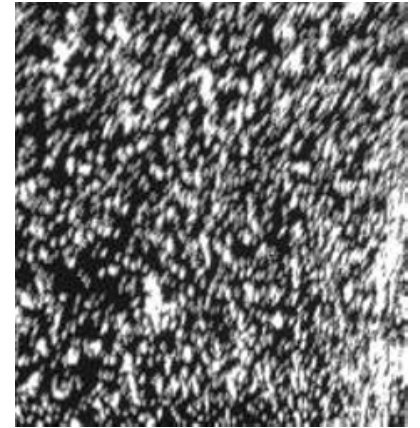

60

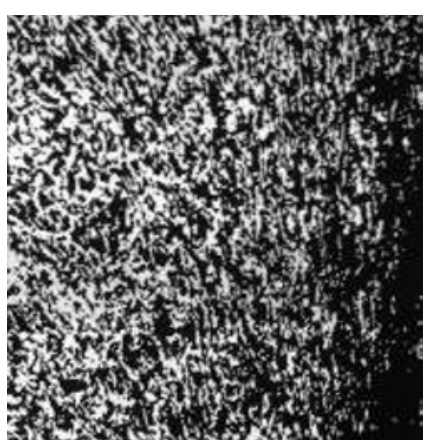

200

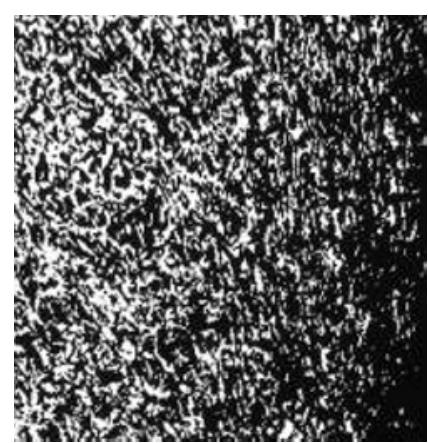

220

Fig. 9. DSNS binary representations (the numbers below the figures are binarization thresholds).

Discontinuity flaws (pores and micro cracks) are not found what indicates that structural transformations are in the limits of two (micro $\div$ meso- or meso- $\div$ macro-) structural levels.

Multifractal analysis of the taget area image shows that the binary picture is characterized by the fractal

dimension $D_{0 f}=1.997$ and the latent order parameter $\delta=0.0256$ (Fig. 10). The dimension value $\left(D_{0 f} \rightarrow 2\right)$ indicates

a strong (greater than in the case of the superplastic behavior of a plane cumulative jet) fragmentation of structural

elements, and a small value of the hidden order $(\delta \rightarrow 0)$ indicates a high probability of structural-phase transformation.

The obtained value of $D_{0 f}$ indicates not only the strongest structure refinement, but also the attainment of a state

close to the extremely fragmented structure. Pores and microcracks were not found, which indicates structure

transformations in the boundaries of two (micro- meso- or meso- macro-) structural levels. After the target structure

reaches the final fragmented state, further scattering of external mechanical energy occurs through a structure-phase transformation into a partial or completely amorphous state.

It is nothing more than equilibrium and ordered state of a compressible (incompressible) viscous fluid. Thus, this phase transformation justifies the use of the hydrodynamic analogy in the theory of high-velocity penetration.

Now let's try to find an answer, what is common between high-speed superplastic deformation and SPD. First, it is the structure refinement. In the case of superplastic deformation, it goes by dynamic polygonization and formation of subgrain structure that is stable up to pre-melting temperatures. And in the case of SPD, it goes by structure fragmentation to the limiting state. Second, in both cases, the latent order parameter has the same low (close to zero) value, which indicates that the structure tends to a structure-phase transformation. In the case of superplastic deformation, this transformation is apparently associated with an abrupt change in the rheological characteristics of the material (which we will touch upon below). In the case of SPD, the structural transformation occurs as a crystal $\rightarrow$ amorphous state transition. 


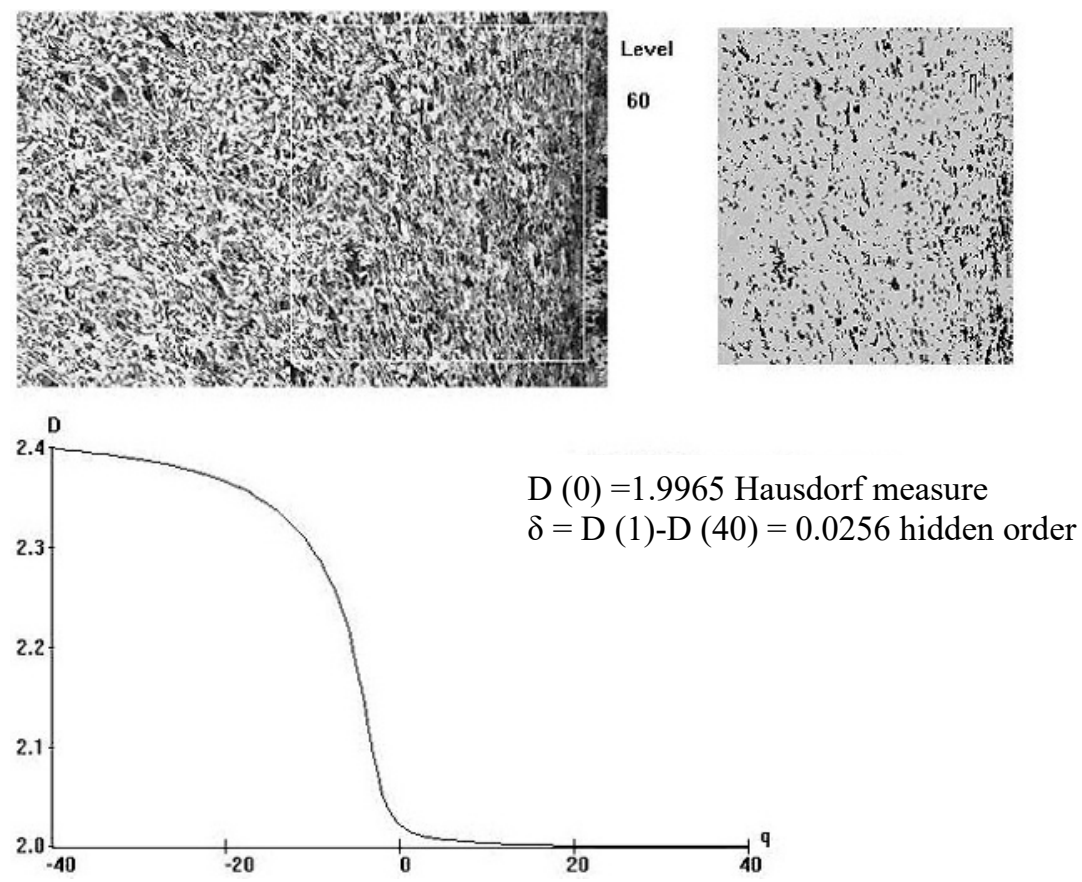

Fig. 10. Images of the multifractal analysis results for the target area on the monitor screen.

What can cause the structural phase transformation in the material of the jet at superplastic behavior? In [17] the author, based on the analysis of the Clausis - Cliperon equation: $\Delta V \frac{d p}{d T}=\frac{\Delta H}{T}$ (here $\Delta V=V_{i}-V_{s}$ - volume transformation effect; $\Delta H=H_{i}-H_{s}$ - heating effect, $V_{i}, H_{i}(i=l, s)$ volume and enthalpy of $i$-phase, $l$ and $s$ denote a liquid and a solid state), showed that a metal system can begin to melt at $T \leq T_{\text {melt }}$ ( $T$ can reach room temperature) under the action of mechanical tension when high tensile stresses are reached. The author believes that the magnitude of such stresses reached $10-20 \mathrm{GPa}$ (in this case, the melting temperature could be below room temperature). Let us estimate the magnitude of the tensile stresses from the elongation of the plane jet. In [18], a formula was derived for estimating the tension $S_{d}$ under tension:

$$
S_{d}=\frac{\rho(\Delta v)^{2}}{12\left(1+\frac{1}{0,8+2,06 \psi}\right) e},
$$

where $\rho=7,8 \cdot 10^{3} \mathrm{~kg} / \mathrm{m}^{3}$ - jet material density; $\Delta v \approx 3500 \mathrm{~m} / \mathrm{s}$ - speeds difference in head and pestle of jet; $\psi=\left(l_{1}-l_{0}\right) / l_{1} \approx 0,8-$ jet reduction $\left(l_{1,0}-\right.$ final and starting jet length); $e=\ln \left(l_{1} / l_{0}\right) \approx 1,61-$ jet true strain. Substituting all the obtained values of the parameters of the jet material into (1), we obtain $S_{d} \approx 3.5 \mathrm{GPa}$. In accordance with [17], such stress significantly reduces the melting temperature, and it can be expected that it will be close to $0.5 \mathrm{Tm}$. 


\section{Conclusions}

1. Methods of light metallography revealed the features in the size, morphology and types of consolidation in the cumulative knife after superplastic deformation and a stationary target after severe plastic deformation at a rate of $10^{4} \mathrm{~s}^{-1}$;

2. In the local (grain-boundary) structure volumes of a plane cumulative jet and target, signs of a structure-phase transition with an assumed change in the aggregation material state in the form of partial or complete amorphization were found.

3. Multifractal parametrization method of structure images should be considered as an experimental technique that makes it possible to supplement the known data on the processes of dynamic high-speed structure formation.

\section{References}

1. V.V. Rybin, Large plastic deformations and destruction of metals (Moscow, Metallurgy, in Russian, 1986)

2. V.A. Pavlov, Phis. Met. Metallogr., 67, 924 (1989) (in Russian)

3. A.M. Glezer, Bull. Russ. Acad. Sci. Phys. 71, 1764 (2007) (in Russian)

4. R.V. Safiullin, Lett. Mater., 2, 32 (2012). (in Russian)

5. V.N. Pereverzentcev, J. Tech. Phys., 66, 140 (1996). (in Russian)

6. U.I. Krasnoshekov, L.K. Kuznetcov, V.N. Pereverzentcev, Dokl. Acad. Nauk USSR, 312, 872 (1990) (in Russian)

7. I.V. Homskaya, V.I. Zeldovich, E.V. Shorokhov, Russ. Metall., N 2, 36 (2009) (in Russian)

8. B.K. Barakhtin, G.G. Savenkov, Voprosy materialovedeniya, 4 (28), 5 (2001) (in Russian)

9. B.K. Barakhtin, V.F. Chashnikov, Voprosy materialovedeniya, 4 (28), 10 (2001) (in Russian)

10. B.K. Barakhtin, A.M. Nemetc, Nanotechnologies Ecology Production 3 (10). 114 (2011) (in Russian)

11. J. Feder, Fractals (New York.: Plenum Press, 1988).

12. G.V. Vstovsky Elements of information physics (Moscow, 2002).

13. A.P. Zhilyaev, A.I. Pshenichnjuk, Superplasticity and grain boundaries in ultra-finegrained materials (M.: 2008) (in Russian)

14. G.G. Savenkov, B.K Barakhtin, K.A. Rudometkin, J. Tech. Phys, 85, 247 (2015) (in Russia)

15. B.K. Barakhtin, G.G. Savenkov, Voprosy materialovedeniya, 4 (64), 60 (2010) (in Russian)

16. B.K Barakhtin., A.A. Prus, G.G. Savenkov, J. Appl. Mech. Tech. Phys., 5, 88 (1989) (in Russian)

17. L.S. Vasiliev, Doct. Diss., Diss... d.f-m.n (Izhevsk: FTI UrO RAS, 2010). (in Russian).

18. G.G Savenkov., N.N. Vasiliev, Probl. of Strength Mater. 10, 47 (1993)/ 